\title{
Genome-wide chromatin footprinting reveals changes in replication origin architecture induced by pre-RC assembly
}

\author{
Jason A. Belsky, ${ }^{1,2}$ Heather K. MacAlpine, ${ }^{1}$ Yoav Lubelsky, ${ }^{1,4}$ Alexander J. Hartemink, ${ }^{2,3}$ \\ and David M. MacAlpine ${ }^{1,2}$ \\ ${ }^{1}$ Department of Pharmacology and Cancer Biology, Duke University Medical Center, Durham, North Carolina 27710, USA; \\ ${ }^{2}$ Program in Computational Biology and Bioinformatics, Duke University, Durham, North Carolina 27708, USA; ${ }^{3}$ Department \\ of Computer Science, Duke University, Durham, North Carolina 27708, USA
}

\begin{abstract}
Start sites of DNA replication are marked by the origin recognition complex (ORC), which coordinates Mcm2-7 helicase loading to form the prereplicative complex (pre-RC). Although pre-RC assembly is well characterized in vitro, the process is poorly understood within the local chromatin environment surrounding replication origins. To reveal how the chromatin architecture modulates origin selection and activation, we "footprinted" nucleosomes, transcription factors, and replication proteins at multiple points during the Saccharomyces cerevisiae cell cycle. Our nucleotide-resolution protein occupancy profiles resolved a precise ORC-dependent footprint at 269 origins in G2. A separate class of inefficient origins exhibited protein occupancy only in G1, suggesting that stable ORC chromatin association in G2 is a determinant of origin efficiency. G1 nucleosome remodeling concomitant with pre-RC assembly expanded the origin nucleosome-free region and enhanced activation efficiency. Finally, the local chromatin environment restricts the loading of the Mcm2-7 double hexamer either upstream of or downstream from the ARS consensus sequence (ACS).
\end{abstract}

[Keywords: DNA replication; origin recognition complex (ORC); chromatin; nucleosome]

Supplemental material is available for this article.

Received June 26, 2014; revised version accepted December 8, 2014.

Eukaryotic DNA replication initiates from multiple locations along each chromosome. These start sites, termed origins of DNA replication, are defined by a primary sequence, essential initiator proteins, and local chromatin structure (Leonard and Méchali 2013). In Saccharomyces cerevisiae, start sites of DNA replication are marked by the ARS consensus sequence (ACS) (Broach et al. 1983; Marahrens and Stillman 1992), a degenerate T-rich motif bound by the heterohexameric origin recognition complex (ORC) (Bell and Stillman 1992). ORC associates with replication origins throughout the cell cycle (Diffley et al. 1994). During G1 and in conjunction with Cdc6 and Cdt1, ORC recruits the $\mathrm{Mcm} 2-7$ helicase to initiation sites, leading to the formation of the prereplicative complex (pre-RC) (Bell and Kaguni 2013). Once the pre-RC is assembled, the origin is licensed for potential activation in S phase (Remus and Diffley 2009). Origin activation is

\footnotetext{
${ }^{4}$ Present address: Department of Biological Regulation, The Weizmann Institute of Science, Rehovot 7610001, Israel

Corresponding author: david.macalpine@duke.edu

Article is online at http://www.genesdev.org/cgi/doi/10.1101/gad.247924.114.
}

temporally regulated, and each origin is characterized by an inherent initiation efficiency (McGuffee et al. 2013) and S-phase activation time (Raghuraman et al. 2001; Yabuki et al. 2002; Müller et al. 2014). Although the precise mechanisms regulating the selection and activation of specific origins in the genome are poorly understood, they are thought to be modulated by epigenetic features and the local chromatin environment (Rhind and Gilbert 2013).

All DNA-templated processes (e.g., replication, recombination, repair, etc.) occur in the context of the surrounding chromatin environment. Chromatin features such as nucleosome positioning can restrict access to particular DNA sequences, limiting which sites are available for protein binding (Bai and Morozov 2010). This likely contributes to the relatively small number of $\sim 220-400$

(c) 2015 Belsky et al. This article is distributed exclusively by Cold Spring Harbor Laboratory Press for the first six months after the full-issue publication date (see http://genesdev.cshlp.org/site/misc/terms.xhtml). After six months, it is available under a Creative Commons License (Attribution-NonCommercial 4.0 International), as described at http:// creativecommons.org/licenses/by-nc/4.0/. 
ORC-binding sites in the $S$. cerevisiae genome despite nearly 10,000 high-quality ACS motif matches (Xu et al. 2006; Eaton et al. 2010). Classic experiments at the ARS1 replication origin demonstrated that artificially positioning a nucleosome over the ACS impaired origin function (Simpson 1990), suggesting that nucleosomes govern ORC accessibility to the ACS. More recent high-throughput nucleosomepositioning assays have established that well-positioned nucleosomes flanking the ACS are a conserved feature of replication origins throughout the genome (Berbenetz et al. 2010; Eaton et al. 2010). In addition, the flanking nucleosome positions may also play an active role in pre-RC formation, as expanding the native nucleosome-free region (NFR) at ARS1 significantly limited pre-RC assembly (Lipford and Bell 2001). These findings demonstrated that chromatin structure significantly impacts ORC binding and pre-RC assembly.

The biochemical cascade of events leading to origin selection and ultimately activation are becoming increasingly well understood. ORC was initially identified as a biochemical activity that "footprinted' the ACS of ARS1 by DNase I hypersensitivity (Bell and Stillman 1992). An extension of this G2 ORC footprint in G1 suggested that pre-RC assembly alters the protein-DNA occupancy at the origin (Diffley et al. 1994; Perkins and Diffley 1998; Speck et al. 2005). Consistent with this change in the molecular architecture of the origin, cryo-electron microscopy (cryo-EM) studies have also revealed a dramatic Cdc6-induced change in the conformation of ORC on template DNA (Sun et al. 2012). In vitro pre-RC assembly experiments demonstrated the capability of loading multiple Mcm2-7 double hexamers that can passively translocate along template DNA prior to activation in S phase (Bowers et al. 2004; Evrin et al. 2009; Remus et al. 2009). Despite our mechanistic understanding of pre-RC assembly and initiation on template DNA in vitro (Speck et al. 2005; Heller et al. 2011; Fernández-Cid et al. 2013; Frigola et al. 2013; Sun et al. 2013), we have little knowledge of how these events are regulated at specific origins in the chromosomal context.

Recent genome-wide data sets, including ORC and Mcm2-7 chromatin immunoprecipitation (ChIP) assays (Wyrick et al. 2001; Xu et al. 2006; Eaton et al. 2010; Szilard et al. 2010), replication timing experiments (Raghuraman et al. 2001; Yabuki et al. 2002; Müller et al. 2014), and recovery of nascent DNA replication intermediates (Feng et al. 2006; Crabbé et al. 2010; McGuffee et al. 2013), have provided a wealth of critical information about the precise location and efficiency of individual replication origins across the genome. However, the rules and chromatin features that govern origin selection and regulation remain elusive. Multiple fundamental questions remain, including the following: (1) Where and how many Mcm2-7 complexes are loaded in relation to ORC-binding sites? (2) Do all origins share the same molecular architecture? (3) How do origin-proximal transcription factors impact origin architecture and regulation? (4) How does origin architecture change throughout the cell cycle? (5) Are specific chromatin features associated with origin efficiency and activation time? Although some of these questions can potentially be addressed biochemically at defined origins in vitro, there will undoubtedly be origin-specific differences that are dependent on the local chromatin environment.

To better understand how the chromatin architecture at individual replication origins impacts origin function, we used micrococcal nuclease (MNase) mapping to comprehensively "footprint" the protein-DNA occupancy across the $S$. cerevisiae genome at multiple points in the cell cycle (Henikoff et al. 2011; Kent et al. 2011). Using this approach, we were able to identify, at nucleotide resolution, DNA fragments protected by nucleosomes, transcription factors, and key components of the pre-RC. Importantly, this technique not only provides significantly higher resolution than other approaches, like ChIP, but is also factor-agnostic and does not rely on enrichment with specific antibodies. We identified 269 origins with an ORC-dependent chromatin footprint. These ORC-dependent footprints were specifically extended during pre-RC assembly in G1 and agreed with previous protection estimates generated from DNase I footprinting at select loci (Diffley et al. 1994; Perkins and Diffley 1998; Speck et al. 2005). Not all origins exhibited the same chromatin architecture; a distinct class of inefficient origins yielded a cell cycle-dependent footprint only in G1, suggesting a transient ORC association at these origins until stabilization in G1. Chromatin remodeling of the nucleosomes flanking the ACS in G1 was a feature of efficient origins. Integrating the nucleotide-resolution "footprints" of origin architecture with ORC and Mcm2-7 ChIP-seq (ChIP coupled with sequencing) provided new insights into Mcm2-7 loading in vivo. Each origin assembles an Mcm2-7 complex either upstream of or downstream from ORC, proximal to the flanking nucleosome, suggesting that, unlike in vitro, only one Mcm2-7 double hexamer is loaded per origin. In summary, MNase mapping provides a critical independent validation of prior ChIP-seq and origin mapping experiments and also yields new mechanistic insights into pre-RC assembly, chromatin dynamics, and origin regulation genome-wide.

\section{Results}

Chromatin footprinting replication origins genome-wide

To interrogate the origin chromatin structure at high resolution, we used a MNase digestion assay that reveals protein occupancy across the genome (Henikoff et al. 2011; Kent et al. 2011). Protein-bound DNA regions are protected from MNase digestion, and DNA fragments $>25$ base pairs $(\mathrm{bp})$ can be recovered for high-throughput paired-end sequencing. We were able to identify distinct regions of DNA protection by nucleosomes $(\sim 150 \mathrm{bp})$ and smaller (25- to 120-bp) protein-DNA interactions (e.g., transcription factors and ORC). MNase mapping provides a comprehensive and unprecedented nucleotide-resolution view of chromatin structure at individual origins throughout the genome.

We first profiled the ORC-dependent chromatin architecture at DNA replication origins in G2, prior to G1 pre$\mathrm{RC}$ assembly. We arrested cells at the G2/M transition by treatment with nocodazole and subjected these cells to 
Belsky et al.

MNase mapping. Chromatin architecture was then visualized at individual replication origins by plotting the chromosomal position of the recovered protected reads as a function of their fragment length. For example, at ARS107, which is situated in the intergenic region between PSK1 and TPD3 on chromosome I, we detected well-positioned nucleosomes, as evidenced by periodic clusters of 150- to 175-bp fragments (Fig. 1A). In addition, we also observed two smaller protection regions $(25-120 \mathrm{bp})$ that indicate specific nonnucleosomal protein-DNA interactions, mapping precisely to the annotated motifs for the ACS and the transcription factor Abflp. Thus, we were able to detect distinct DNA-binding events in a comprehensive and factoragnostic manner.

To confirm that the 25- to 120-bp fragments at the ACS of ARS107 originated from and were dependent on ORC occupancy, we evaluated the chromatin architecture in the temperature-sensitive orc1-161 mutant. In the orc1161 mutant, ORC can no longer associate with DNA at the nonpermissive temperature $\left(37^{\circ} \mathrm{C}\right)$ (Aparicio et al. 1997). MNase mapping revealed that the chromatin protection footprint at the ACS of $A R S 107$ was completely lost (Fig. 1A, bottom panel); in contrast, the chromatin occupancy at the annotated Abf1-binding site was unaffected. Loss of ORC at the ACS also resulted in nucleosome encroachment over the replication origin. This chromatin reorganization impacted the nucleosome positioning within the PSK1 gene body but not the TPD3 gene, likely due to the barrier effects of Abflp (Mavrich et al. 2008; Zhang et al. 2009). Therefore, MNase mapping can capture dynamic chromatin changes that occur when protein-DNA binding is altered.

The ORC-dependent chromatin architecture that we observed at $A R S 107$ was representative of origins throughout the genome. We generated aggregate wild-type and orc1161 chromatin occupancy profiles from replication origins identified in a prior ORC ChIP-seq study (Eaton et al. 2010) by aligning each origin to the $5^{\prime}$ position of the ACS motif (Fig. 1B). To ensure directional consistency, we oriented the ACS at every replication origin relative to the T-rich strand. In describing chromatin features relative to the ACS, upstream and downstream correspond to the $5^{\prime}$ and $3^{\prime}$ side of the T-rich ACS strand, respectively. As previously described in wild type (Eaton et al. 2010), MNase mapping of origins revealed well-positioned nucleosomes around the
A

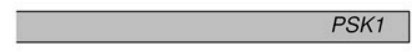

WT

orc1-161

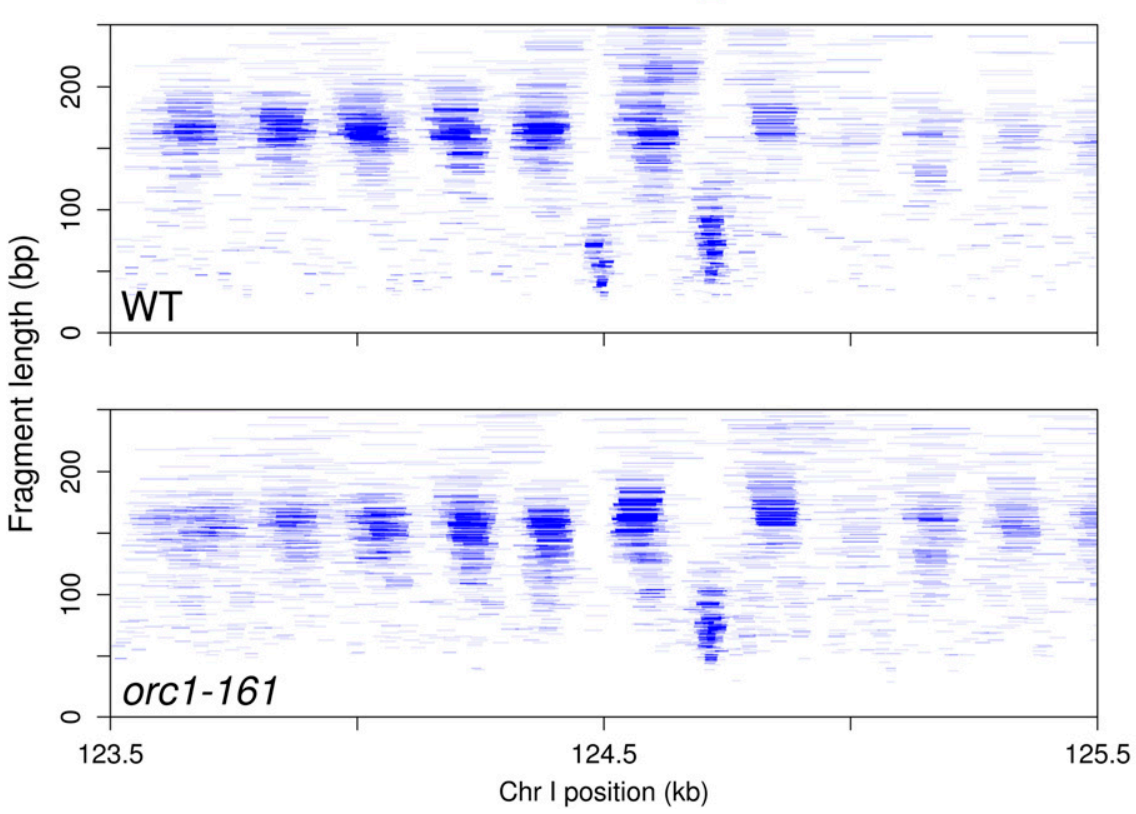

ช

TPD3
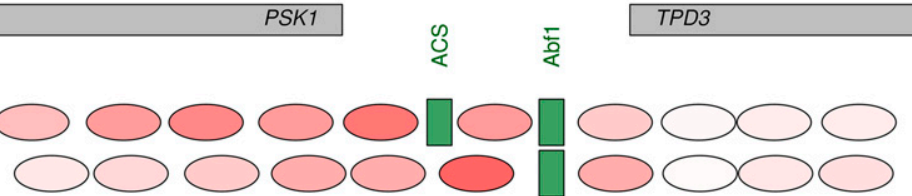

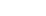

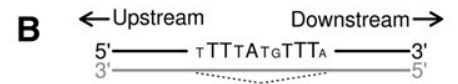

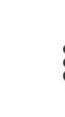
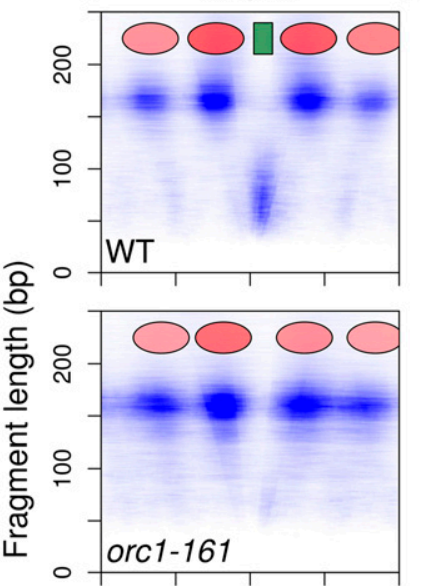

WT

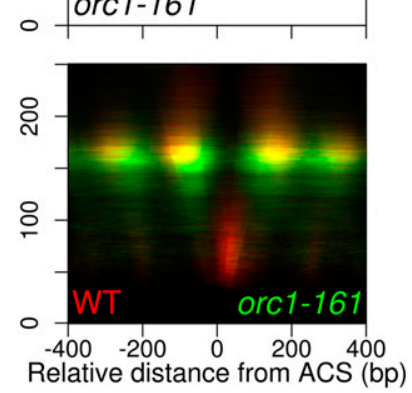

Figure 1. Identification of an ORC-dependent footprint by MNase mapping. (A) Wild-type (WT) and orc1-161 G2 chromatin profiles at the ARS107 locus. Recovered and sequenced MNase fragments are displayed with their chromosomal coordinate on the $X$-axis and their fragment length on the $Y$-axis. Clusters of reads in the $\sim 150$ - to 175 -bp range denote protection by nucleosomes. Consensus nucleosome positions are represented by red ovals, and the shading corresponds to the total occupancy of a particular nucleosome. Smaller fragments (25-120 bp) indicate protein-DNA occupancy from sequence-specific DNA-binding factors (green rectangles). Annotated DNA-binding motifs (Abf1 and ACS) are indicated in green. (B) Aggregate chromatin profiles from a previously characterized replication origin data set (ORC-ACS) (Eaton et al. 2010), with all origins oriented by the T-rich ACS strand. The top and middle panels are derived from wild-type and orc1-161 MNase digestions, respectively. The bottom panel represents a merged overlay of wild type (red) and orc1-161 (green). 
ACS (Fig. 1B, top panel). Interestingly, the bulk of the DNA protection from small fragments occurred in the middle of the NFR, suggesting that the ORC footprint extends downstream from the ACS. In contrast, DNA protection at the ACS was completely lost in the orc1-161 mutant at the nonpermissive temperature (Fig. 1B, middle panel). Similar to $A R S 107$, loss of ORC resulted in the encroachment of nucleosomes over the ACS, highlighted by a high concentration of 150 - to $175-\mathrm{bp}$ reads closer to the ACS in the orc1-161 mutant (Fig. 1B, bottom panel). Importantly, we did not observe any significant differences in protection of DNA features outside of replication origins (Supplemental Fig. S1A,B).

The MNase assay provides an independent and highresolution method to assess the chromatin occupancy at all potential replication origins genome-wide. We compared the G2 protection of the ACS in wild type (Fig. 2A) and orc1-161 (Fig. 2B) at nearly 800 putative origins that exhibited ARS function in a plasmid-based assay (Nieduszynski et al. 2007). By focusing on only the 25- to 120-bp DNA fragments, we identified 269 origins exhibiting an ORC-dependent footprint in G2 (Fig. 2C). The chromatin occupancy differences were restricted to the region surrounding the ACS, demonstrating the precision of this approach (Fig. 2C; Supplemental Fig. S1C). These 269 origins were also highly enriched in ORC ChIP-seq signal (Fig. 2D), thus providing independent verification that these sites are likely occupied by ORC in vivo. We conclude that the in vivo chromatin environment restricts ORC binding to only a subset of potential replication origins.

\section{ORC only stably associates with a subset of functional origins in G2}

As the cell cycle progresses through G1, ORC, together with Cdc6 and Cdt1, directs the loading of the Mcm2-7 helicase at replication sites to form the pre-RC (Bell and Kaguni 2013). Although pre-RC formation has been extensively studied in vitro (Speck et al. 2005; Heller et al. 2011; Fernández-Cid et al. 2013; Frigola et al. 2013; Sun et al. 2013), footprinting studies have been limited to only a few origins (Diffley et al. 1994; Perkins and Diffley 1998; Speck et al. 2005). It remains unclear how the local chromatin architecture and origin-specific protein-DNA interactions impact pre-RC assembly and subsequent DNA replication initiation.

To address these questions, we evaluated the originspecific chromatin architecture during ORC binding in G2/M (nocodazole arrest) and following pre-RC assembly in $\mathrm{Gl}$ ( $\alpha$-factor arrest). We observed a small but significant expansion in the ORC-dependent footprint from G2 to G1 (Fig. 3A; Supplemental Fig. S2A). Importantly, this footprint expansion was specific to replication origins, as other transcription factor-binding sites did not yield any protection differences between G2 and G1 (Supplemental Fig. S2B). To test whether this expansion was dependent on pre-RC assembly, we used a temperature-sensitive

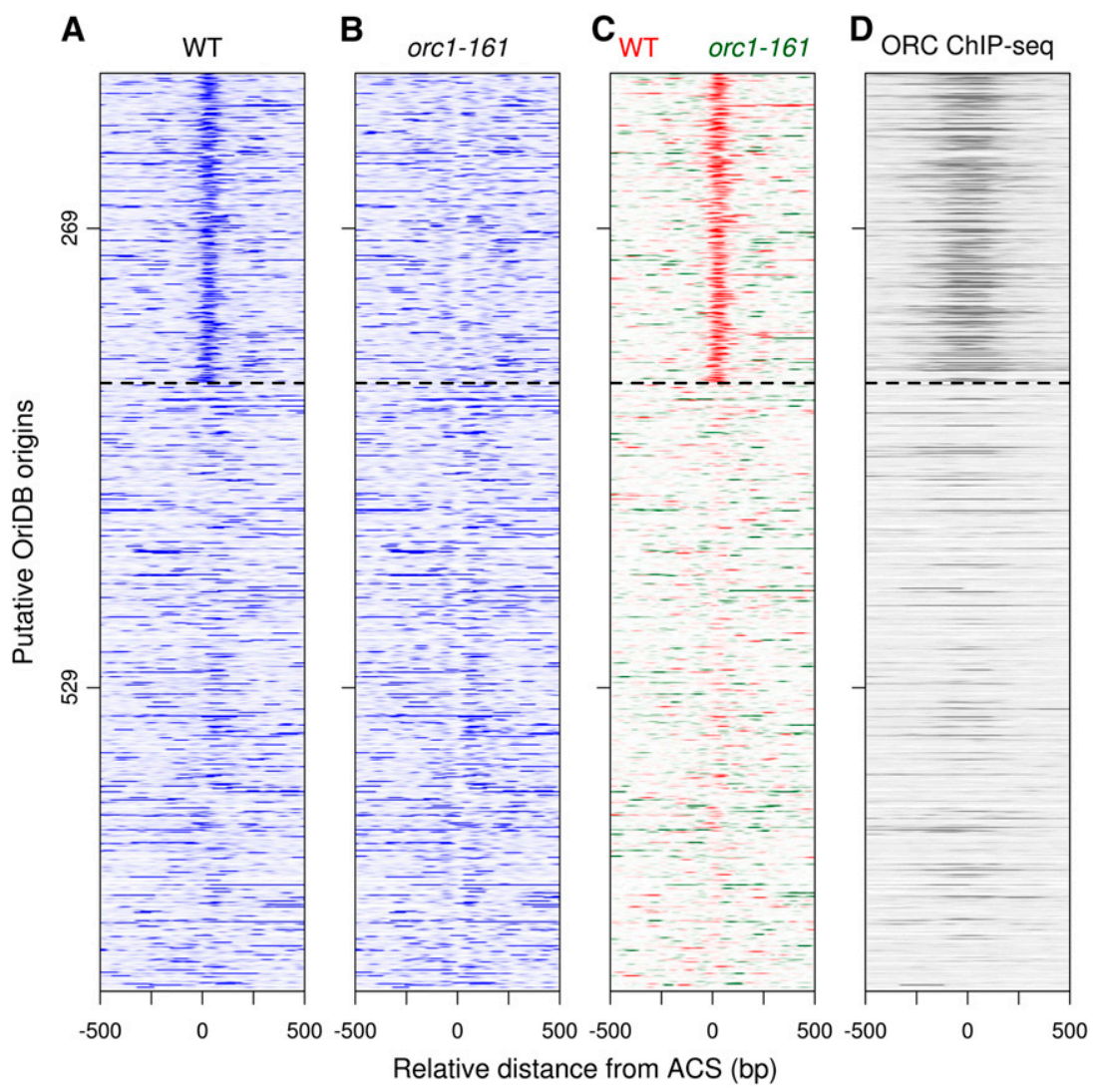

Figure 2. ORC only associates with a subset of putative origins. Small fragments $(25-120$ bp) surrounding the ACS at each of the 798 putative (exhibiting ARS function on a plasmid) OriDB origins (Nieduszynski et al. 2007) were condensed into single footprint signals for wild type (WT) $(A)$ and orc1-161 (B). Each row in the heat map represents an individual origin oriented by its T-rich ACS strand as in Figure 1B. (C) The orc1-161 (green) small fragment signal was subtracted from the wild-type (red) small fragment signal to yield a difference heat map. An ORC-dependent footprint was detected at 269 origins (top subset). (D) ORC ChIP-seq data from asynchronous cells largely overlapped with replication origins containing an ORC-dependent footprint (top subset). 
A

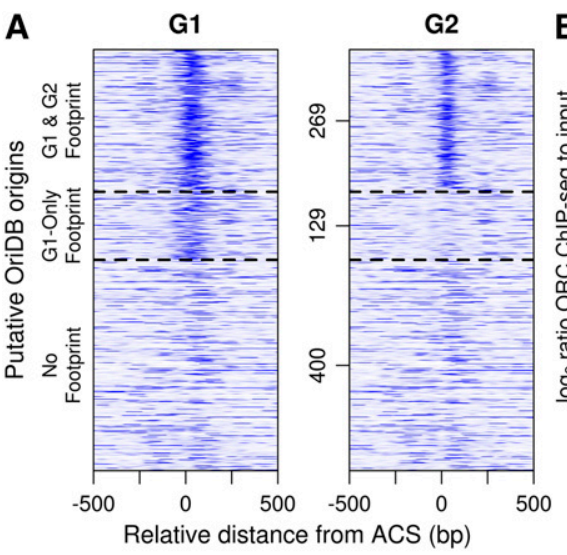

C
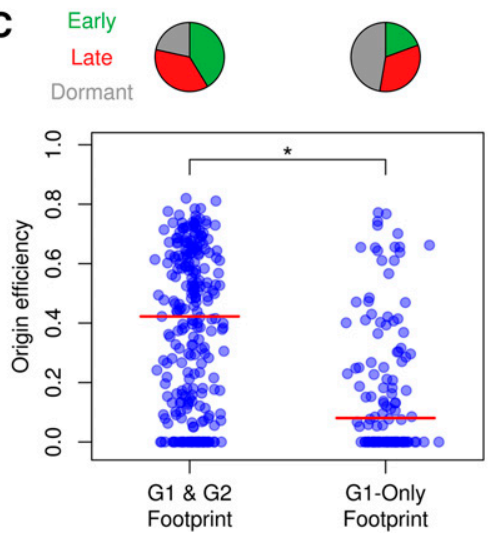

B
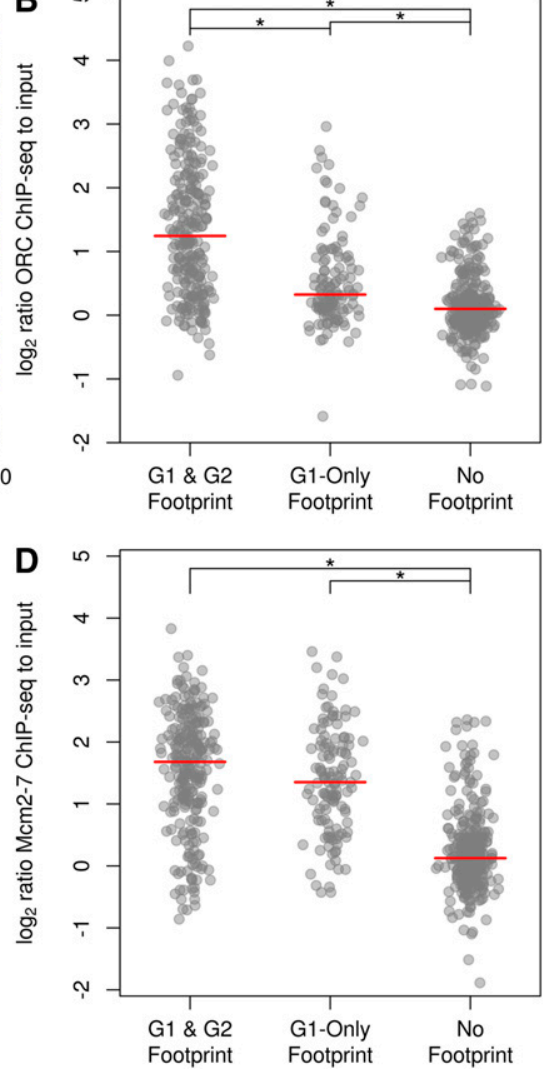

Figure 3. ORC-chromatin association in G2 is a determinant of origin efficiency. $(A)$ Heat map of small fragment (25-120 bp) footprint occupancy surrounding the ACS at each putative OriDB origin (Nieduszynski et al. 2007) for G1-arrested (left panel) and G2-arrested (right panel) cells. ORC-dependent footprint origins displayed an increased occupancy and wider protection region in G1 (G1 \& G2 Footprint). A separate origin class exhibited protection of the ACS region specifically in G1 (G1-Only Footprint). (B) Distribution of asynchronous ORC ChIP-seq enrichment for each putative origin. ORC ChIP-seq signal was significantly greater in the G1 and G2 footprint class compared with either the G1-only footprint or no footprint classes (Wilcoxon test, $P<6.80 \times 10^{-13}$ and $P<$ $4.81 \times 10^{-43}$, respectively). A smaller yet significant difference was also detected in the G1-only footprint class relative to the no footprint class $\left(P<1.05 \times 10^{-7}\right)$. $(C)$ Origin activation and efficiency for the G1 and G2 footprint and G1-only footprint classes. The G1 and G2 footprint class was more efficient $\left(P<1.61 \times 10^{-11}\right)$ and earlier activating $\left(\chi^{2}\right.$ test, $\left.P<1.20 \times 10^{-7}\right)$ than the G1-only footprint class. $(D)$ Distribution of G1 Mcm2-7 ChIP-seq enrichment for each putative origin. The G1 and G2 footprint and G1-only footprint classes showed no significant difference in Mcm2-7 signal (Wilcoxon test, $P<0.035$ ), but each contained greater $\mathrm{Mcm} 2-7$ signal than the no footprint class $\left(P<3.48 \times 10^{-43}\right.$ and $P<3.93 \times 10^{-28}$, respectively).

allele of CDC6 (cdc6-1) (Hartwell et al. 1973) to abrogate pre-RC assembly (Supplemental Fig. S2C). In the absence of Cdc6 activity, we found that the expansion of the G1 footprint at origins was lost, and the chromatin more closely resembled the G2 state (Supplemental Fig. S2A). The expanded G1 footprint may reflect increased protection conferred by the ORC-Cdc 6 complex or the Mcm2-7 double hexamer.

A subset of origins exhibited a G1 chromatin occupancy at the ACS yet lacked a G2 ORC-dependent footprint (Fig. $3 \mathrm{~A})$. The absence of a G2 ORC-dependent footprint suggested that ORC either binds transiently in G2 or does not associate with these origins until G1 and pre-RC assembly. Not surprisingly, ORC ChIP-seq signal in asynchronous cells was substantially less at these origins relative to the sites exhibiting a G2 ORC-dependent footprint (Fig. 3B). Impaired ORC binding is not likely to be caused by a weak ACS motif, as both origin classes yielded nearly identical sequence motifs (Supplemental Fig. S3).

We next asked whether the absence of an ORCdependent footprint in G2 had any functional consequences. To assess origin function, we used the distribution of Okazaki fragments (McGuffee et al. 2013) and BrdU incorporation during a hydroxyurea arrest as proxies for origin efficiency and origin activation time, respectively. We found that origins not harboring a G2 ORC-dependent footprint are much more likely to be dormant, inefficient, and late-firing (Fig. 3C). However, this defect in origin activation does not appear to be due to diminished pre-RC assembly, as there is no significant reduction in Mcm2-7 loading as determined by ChIP-seq enrichment for this origin class (Fig. 3D). Together, these data suggest that stable ORC association in G2 is a determinant for efficient or early activating replication origins.

\section{Nucleosome dynamics in G1 promote origin activation}

A defining feature of replication origins is a well-established NFR flanked by nucleosomes asymmetrically positioned around the ACS (Berbenetz et al. 2010; Eaton et al. 2010). Disruption of this optimal nucleosome positioning, such as artificial movement of the downstream nucleosome at ARS1, adversely affects replication function (Lipford and Bell 2001). Although the sequences at the replication origin are inherently nucleosome-disfavoring, ORC binding at the ACS and an ATP-dependent chromatin remodeling activity are necessary and sufficient to establish this nucleosome organization in vitro (Eaton et al. 2010). However, it is currently unknown whether pre-RC assembly or replication initiation requires additional chromatin remodeling.

To address this question, we compared the G1 and G2 flanking nucleosome positions from all origins harboring a chromatin footprint in G1. We constructed G1 and G2 
nucleosome profiles from the midpoint positions of all 150- to 175-bp DNA fragments surrounding replication origins. To focus on cell cycle-dependent differences in nucleosome positioning, we plotted the log ratio of G1 to G2 nucleosome density as a heat map (Fig. 4A). We observed three different cell cycle-dependent nucleosome configurations. We found that $41 \%$ of the origins maintained static nucleosome positioning between G1 and G2; in contrast, the other two origin configurations exhibited expanded NFRs in G1 by shifting either the upstream (26\%) or downstream $(33 \%)$ nucleosome but not both. Average G1 and G2 nucleosome density plots exhibited wellpositioned nucleosomes within each class (Supplemental Fig. S4A). We conclude that a subset of origin-flanking nucleosomes undergo cell cycle-dependent chromatin remodeling immediately prior to or during pre-RC assembly.

We hypothesized that the chromatin remodeling events observed at a subset of origins may be dependent on pre$\mathrm{RC}$ assembly. To test this hypothesis, we blocked pre-RC formation by using a temperature-sensitive $c d c 6-1$ mutant and assayed nucleosome positioning in $\alpha$-factor G1-arrested cells at the restrictive temperature. In the absence of pre-RC formation, all origins now maintained their consensus G2 nucleosome positions (Fig. 4B; Supplemental Fig. S4B), thus establishing a link between pre-RC assembly and nucleosome remodeling at a subset of replication origins.

Presumably, the chromatin remodeling and subsequent enlargement of the NFR directs pre-RC assembly and Mcm2-7 helicase loading. We re-examined the expansion of the ORC-dependent footprint in G1 for each class of observed nucleosome movements: upstream, downstream, and static. We found that the footprint expansion at individual origins was predictive for each class of nucleosome movements (Fig. 4C). Specifically, an upstream expansion of the origin-specific footprint was associated with the movement of the upstream nucleosome, and vice versa for the downstream expansion. Not surprisingly, limited expansion of the footprint was observed for the static class of nucleosome movements. Finally, as with the nucleosome remodeling, the footprint expansion in G1 was dependent on Cde6 activity.

The expanded G1 footprint may reflect increased protection conferred by the ORC-Cdc6 complex, which in vitro extends the ORC footprint by $\sim 30$ bp (Speck et al. 2005), or the Mcm2-7 double hexamer, whose $230 \AA$ A central channel protects $\sim 68 \mathrm{bp}$ of DNA (Remus et al. 2009). As we observed an $\sim 25$-bp average upstream or downstream expansion (Fig. 4C), we most likely recovered the ORCCdc6 complex. We speculate that the Mcm2-7 complex, which can freely slide on DNA templates (Evrin et al. 2009; Remus et al. 2009), translocates off the MNase-digested DNA due to the limited efficiency of formaldehyde crosslinking (Schmiedeberg et al. 2009).

Is nucleosome remodeling important for origin function? We examined origin efficiency and activation time for the three classes of nucleosome movements (upstream, downstream, and static) (Fig. 4D). We found that origins that underwent nucleosome remodeling in G1 were
A

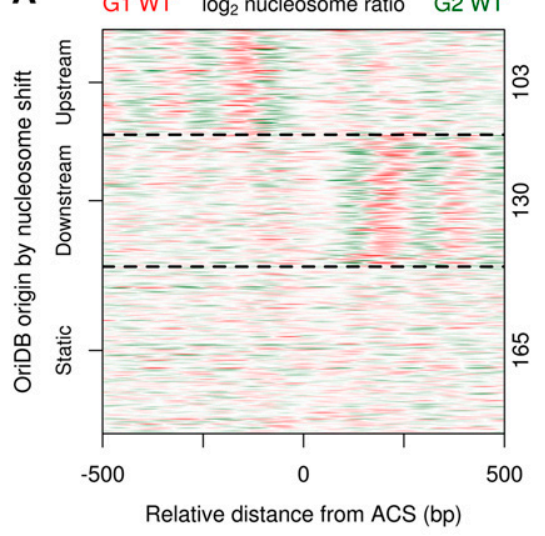

B $\quad \begin{array}{lll}\text { G1 } & \log _{2} \text { nucleosome ratio } & \text { G2 WT }\end{array}$

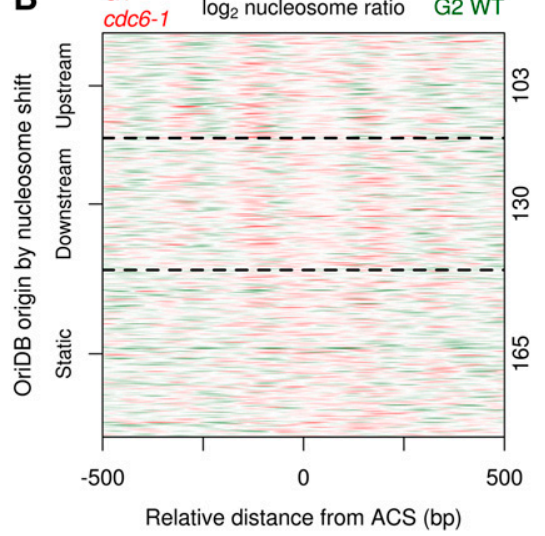

C - G1 WT - G1 $\mathrm{Wdc}_{\mathrm{W}-1}-\mathrm{G} 2 \mathrm{WT}$ Footprint density by nucleosome shift

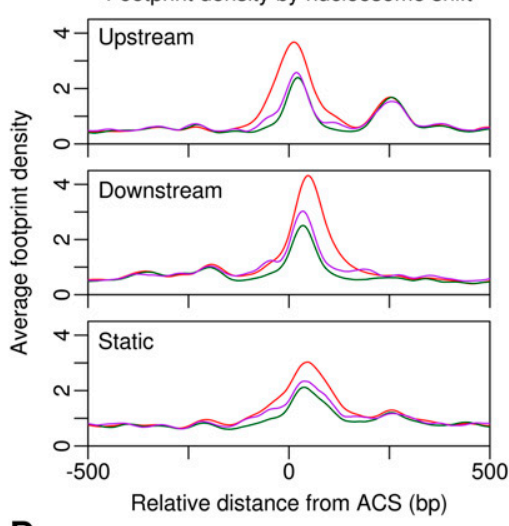

D
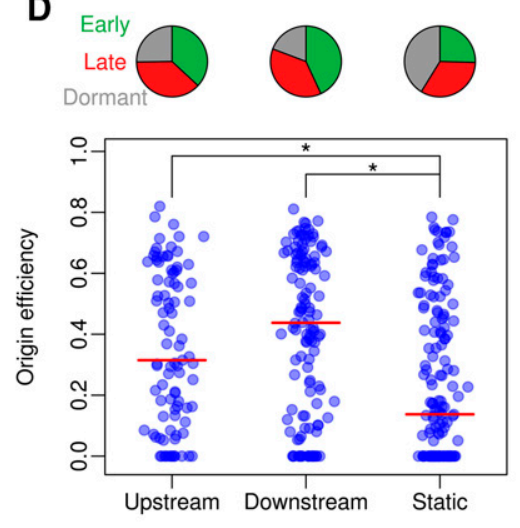

Figure 4. Cell cycle-dependent nucleosome dynamics promote origin efficiency. $(A)$ Heat map representing the $\log _{2}$ ratio of G1 wildtype (WT) (red) to G2 wild-type (green) nucleosome density. Three classes of nucleosome movement (upstream, downstream, and static) were identified. $(B)$ Heat map representing the $\log _{2}$ ratio of G1 cdc6-1 (red) to G2 wild-type (green) nucleosome density, ordered as in $A$. (C) Average small fragment (25-120 bp) footprint occupancy surrounding the ACS for each class of nucleosome positioning in G1 wild type (red), G1 cdc6-1 (purple), and G2 wild type (green). (D) Origin activation and efficiency for each class of nucleosome positioning. Both the upstream and downstream shifting nucleosome classes were more efficient than the static nucleosome class (Wilcoxon test, upstream vs. static, $P<1.42 \times$ $10^{-4}$; downstream vs. static, $P<2.03 \times 10^{-7}$; upstream vs. downstream, $P<0.134)$ and earlier activating $\left(\chi^{2}\right.$ test, upstream vs. static, $P<0.0206$; downstream vs. static, $P<1.04 \times$ $10^{-4}$; upstream vs. downstream, $P<0.474$ ). 
significantly more efficient. This increased origin activation may be explained by additional pre-RC assembly in the presence of dynamic nucleosomes. We examined Mcm2-7 ChIP-seq enrichment within each replication origin class (Supplemental Fig. S4C). We found that Mcm2-7 loading was significantly impaired in the static class, although clearly not reduced to background levels. These results suggest that G1 nucleosome movement promotes origin activation at the pre-RC assembly step; however, given that Mcm2-7 levels are not reduced to background levels, we cannot rule out the possibility that the nucleosome remodeling facilitates steps subsequent to pre-RC assembly, including origin unwinding, helicase activation, or DNA polymerase recruitment.

\section{Mcm2-7 associates with one origin-dependent flanking nucleosome}

Our chromatin structure analysis using MNase digestion revealed cell cycle-dependent changes in origin architecture but did not provide insight into where the $\mathrm{Mcm} 2-7$ complex was loaded relative to ORC. Given the large NFR downstream from the ACS, we hypothesized that Mcm2-7 double-hexamer loading is confined to the unoccupied gap between ORC and the downstream nucleosome. To test this hypothesis, we used ChIP-seq to precisely localize ORC and $\mathrm{Mcm} 2-7$ relative to the chromatin features that we characterized using MNase mapping (Fig. 5A). We first identified the peaks from ORC (Fig. 5A, green) or Mcm2-7 (Fig. 5A, purple) ChIP-seq experiments. Relative to the chromatin structure revealed by MNase mapping (Fig. 5A, gray), we found that the ORC ChIP-seq peaks were concentrated in the NFR and coincided with the ACS. In contrast, we found that the Mcm2-7 ChIP-seq peaks were located either upstream of or downstream from the ORC footprint and appeared to overlap with the flanking nucleosome positions (only one peak was associated with each origin). The apparent occupancy of the same DNA sequence by both the histone octamer and Mcm2-7 suggested the following models: (1) A dynamic competition between Mcm2-7 and nucleosomes resulted in stochastic occupancy of the same position. (2) The Mcm2-7 complex protected an $\sim 150$-bp region of DNA mimicking a histone octamer. (3) The Mcm2-7 complex was immediately adjacent to or in close contact with the nucleosome, and they coimmunoprecipitated as a complex.

We first considered stochastic model 1 , in which the origin-flanking DNA is occupied by either a histone octamer or the Mcm2-7 complex. If Mcm2-7 loading resulted in nucleosome eviction, then replacement of a nucleosome by a Mcm2-7 double hexamer should substantially reduce the G1 nucleosome occupancy. We classified origins into two groups, upstream and downstream, based on the Mcm2-7 ChIP-seq enrichment at either flanking nucleosome and compared the G1 and G2 nucleosome occupancy. However, we detected no cell cycle-dependent nucleosome density differences at the Mcm2-7 enrichment location (Supplemental Fig. S5A,B). Furthermore, both origin-flanking nucleosomes exhibited similar occupancy levels within each group. Combined, these re- sults fail to support a model of stochastic nucleosome occupancy at Mcm2-7-binding locations. We next considered model 2, in which the Mcm2-7 complex was protecting an $\sim 150$-bp fragment of DNA analogous to the protection conferred by a histone octamer. However, it seemed unlikely that a double hexamer of $\mathrm{Mcm} 2-7$ whose $230 \AA$ central channel is predicted to only protect $\sim 68$ bp of DNA (Remus et al. 2009) would be sufficient to generate an $\sim 150$-bp occupancy footprint. Furthermore, both upstream and downstream Mcm2-7 ChIP enrichment groups revealed no differences in the length distributions of MNase-protected fragments (Supplemental Fig. S5C), strongly suggesting that the MNase-protected occupancy is due to a histone octamer.

Finally, we considered model 3, in which the Mcm2-7 double hexamer may be immediately adjacent to or in complex with the flanking nucleosomes. We analyzed the distribution of the forward (upstream) and reverse (downstream) reads that are generated from every sequenced immunoprecipitated fragment within each Mcm2-7 ChIPseq enrichment group (Fig. 5B). The forward (Fig. 5B, red) and reverse (Fig. 5B, green) read positions demarcate the ChIP enrichment boundaries and precisely identify the protein-binding region. When we plotted the forward and reverse reads for the ORC-associated fragments (Fig. 5C, middle left panel), regardless of whether the origin loaded Mcm2-7 upstream or downstream, we found that the peaks of reverse reads (Fig. 5C, green) were located in the NFR downstream from the ACS (Fig. 5C, top left and bottom left panels). The forward ORC reads (Fig. 5C, red) exhibited a bimodal peak, indicating that the fragmentation upstream of ORC occurred either between ORC and the adjacent nucleosome or upstream of the nucleosome. As this pattern was detected only at the upstream nucleosome, it may indicate an interaction between ORC and the adjacent nucleosome, potentially mediated by the BAH domain of ORC1 (Onishi et al. 2007). Unlike with ORC, no putative nucleosome interactions were observed for the transcription factor Abflp by ChIP-seq (Supplemental Fig. S6).

In contrast, the forward and reverse peak positions of the Mcm2-7 ChIP-seq reads encompassed the flanking nucleosomes (Fig. 5C, middle right panel). In the cases where $\mathrm{Mcm} 2-7$ was loaded upstream of the origin, we found that the forward reads (Fig. 5C, red) coincided with the left edge of the nucleosome, and the reverse reads (Fig. 5C, green) were within the NFR (Fig. 5C, top right panel). Similarly, origins loading Mcm2-7 downstream yielded a complementary pattern in the forward and reverse read distributions flanking the downstream nucleosome (Fig. $5 \mathrm{C}$, bottom right panel). Importantly, the total distance between the peaks of forward and reverse reads was $\sim 210$ $\mathrm{bp}$, almost exactly the expected width of one nucleosome ( $150 \mathrm{bp}$ ) and Mcm2-7 double hexamer ( 68 bp). Finally, we found no differences in origin activation or efficiency that could be attributed to Mcm2-7 loading upstream of or downstream from the ACS (Supplemental Fig. S5D). Similarly, we were unable to link the Mcm2-7 loading side with the directional Cdc6-dependent ORC footprint expansion and nucleosome shift. We conclude that origin 
A

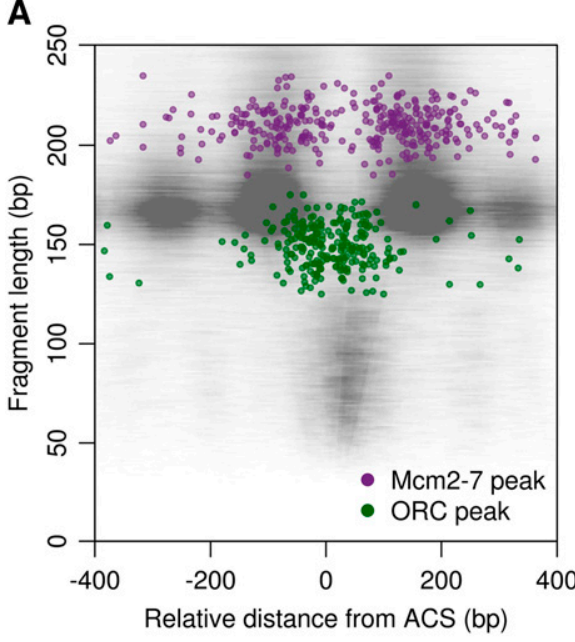

B

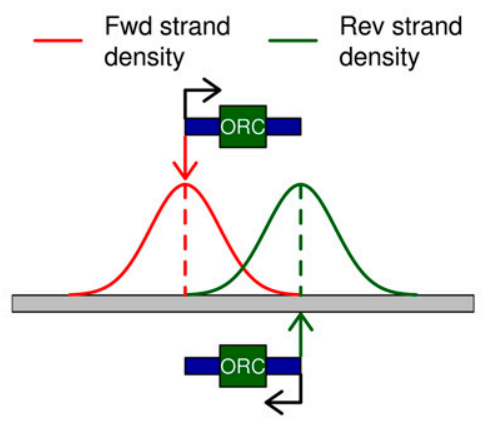

C
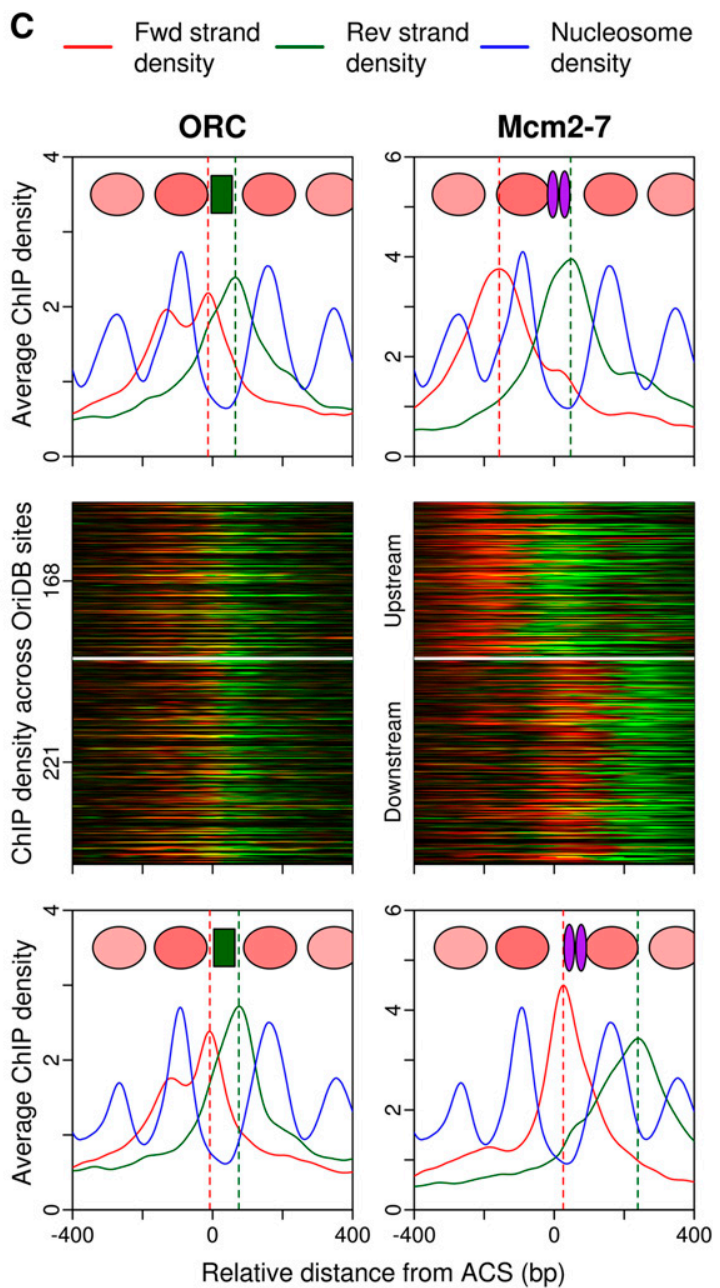

Figure 5. Mcm2-7 loads either upstream of or downstream from ORC. (A) Locations of ORC (green) and Mcm2-7 (purple) ChIP-seq peaks relative to the G1 MNase chromatin data (gray) surrounding origins. For each of the 398 origins with a G1 footprint, 257 asynchronous ORC and 332 G1 Mcm2-7 peaks were identified. The ChIP-seq peak position relative to the ACS was plotted on the $X$ axis, and random noise was applied along the $Y$-axis to spread the data points. $(B)$ Hypothetical distribution of ChIP-seq forward (red) and reverse (green) reads representing chromatin fragmentation patterns around a specific DNA-binding factor. The most likely protein-binding region can be inferred as the area between the two read distributions. $(C)$ Analysis of chromatin fragmentation patterns resulting from ORC (left) and Mcm2-7 (right) ChIP-seq. The 389 origins with sufficient Mcm2-7 ChIP-seq signal were grouped into two classes (upstream and downstream) based on the enrichment signal relative to the origin-flanking nucleosomes. Heat maps (separated by class) represent the distributions of forward (red) and reverse (green) reads at each origin relative to the ACS for ORC (left) and Mcm2-7 (right). Origins have the same ordering in both heat maps. The top and bottom panels represent the average forward and reverse ORC and Mcm2-7 ChIP-seq densities within each class. The most probable fragmentation boundaries are designated by red and green dotted lines for forward and reverse reads, respectively. The average G1 nucleosome density (blue) for each class is superimposed on each plot, and inferred nucleosome positions are depicted as red-shaded ovals.

licensing can yield at least two pre-RC configurations in which Mcm2-7 has been preferentially loaded adjacent to, and likely in complex with, either the upstream or downstream nucleosome.

\section{Discussion}

Using MNase mapping, we comprehensively profiled the cell cycle-dependent local chromatin environment surrounding DNA replication origins. This assay maps DNAbinding protein occupancy across the genome in a costeffective and factor-agnostic manner. We precisely identi- fied 269 ORC-dependent footprints in G2 and found that only a subset of all putative origins (identified in a plasmidbased assay) harbored an ORC-dependent footprint in vivo. We also found that differences in origin efficiency were associated with cell cycle-dependent changes in both origin chromatin architecture and ORC association. Chromatin remodeling of the flanking nucleosomes between G2 and G1 was linked to active and efficient replication origins and was dependent on Cdc6. Finally, integration of the origin chromatin occupancy profiles with ORC and Mcm2-7 ChIP-seq revealed that the Mcm2-7 double hexamer preferentially loads either upstream of or down- 
stream from ORC, adjacent to the flanking nucleosome. These data provide new insights into how local chromatin architecture modulates origin selection and function.

Genome-wide approaches based on ChIP-chip and ChIP-seq targeted against pre-RC components have been used by multiple groups to pinpoint origins in their chromosomal context (Wyrick et al. 2001; Xu et al. 2006; Eaton et al. 2010; Szilard et al. 2010). While ChIP has the potential for increased sensitivity (due to the biochemical enrichment by immunoprecipitation), it is also susceptible to nonspecific interactions (Teytelman et al. 2013) and epitope masking (Aparicio et al. 1997), which may result in the detection of false positives and negatives, respectively. Indeed, the number of ORC-binding sites in yeast varies from conservative estimates of 220 to potentially $>400$, and a number of putative origins contain Mcm2-7 ChIP enrichment without any ORC ChIP signal (Wyrick et al. 2001; Xu et al. 2006; Eaton et al. 2010). In comparison with our previously identified 253 conservative ORC ChIP-seq peaks (Eaton et al. 2010), 179 are present in our G1 and G2 footprint data set (70.8\% overlap). The 74 ORC sites identified by the ChIP assay and not MNase footprinting may represent weak and/or transient binding events or false positives from nonspecific interactions (Teytelman et al. 2013). Conversely, the MNase assay detected 90 ORC-dependent footprints in putative origins that were missed by the ChIP-seq assay. As these origins all harbored an ORC-dependent footprint, it is consistent with prior reports indicating that a significant fraction of ORC-binding sites may be missed by ChIP-seq due to epitope masking (Wyrick et al. 2001; Xu et al. 2006). In summary, although the MNase-based assay may miss weak or transient interactions, it provides increased resolution compared with ChIP approaches, is not sensitive to epitope masking, and serves as an important independent validation of origin location throughout the genome.

A subset of origins had no discernable ORC-dependent occupancy in G2 yet exhibited a protected footprint in G1 (Fig. 3A). Since the MNase chromatin assay may not detect transient interactions, we propose that ORC may only weakly or dynamically associate with these origins until pre-RC assembly in G1 stabilizes ORC binding. These origins have limited G2 ORC residence, supported by a significantly reduced ORC ChIP signal from an asynchronous cell population (Fig. 3B). Origins that failed to associate with ORC in G2 had a dramatically reduced origin efficiency (Fig. 3C), reminiscent of prior observations in Schizosaccharomyces pombe (Wu and Nurse 2009), which linked time of ORC association in mitosis with origin function. We propose that constitutive ORC association with DNA is one determinant of origin efficiency, and as cells enter G1, ORC-associated origins will immediately commence with pre-RC assembly; however, pre-RC formation will likely be delayed for those origins lacking a stable ORC-DNA interaction prior to mitosis. Even though the final levels of Mcm2-7 loading appear unaffected (Fig. 3D), this pre-RC assembly delay possibly handicaps recruitment of Cdc45, Dbf4, and other limiting replication initiation factors (Aparicio et al. 1997; Pasero et al. 1999; Mantiero et al. 2011; Tanaka et al. 2011).
Pre-RC formation in G1 leads to Cdc6-dependent chromatin remodeling events that subsequently impact origin efficiency. We identified three cell cycle-dependent nucleosome configurations at replication origins in G1 (Fig. 4A). The first two classes exhibited either upstream or downstream (but not both) nucleosome movement away from the ACS-oriented origin, while a third comparably inefficient static class did not reposition either flanking nucleosome. We considered the straightforward hypothesis that G1 nucleosome movement increases the origin NFR and thus provides more space for Mcm2-7 loading. However, even though the static class had significantly reduced Mcm2-7 levels, they were still above background levels of Mcm2-7 loading (Supplemental Fig. S4C). We propose that in addition to promoting Mcm2-7 loading, origin nucleosome remodeling may also establish a more favorable chromatin environment for subsequent replication initiation events, including Cdc45 and GINS recruitment (Ilves et al. 2010), DNA unwinding, or DNA polymerase loading. Since precise nucleosome positioning is important for Mcm2-7 assembly (Lipford and Bell 2001), nucleosome displacement is possibly regulated by specific chromatin remodelers like Ino80 or Isw2. These remodelers have already been associated with replication forks (Shimada et al. 2008; Vincent et al. 2008), but our results suggest a potentially new role for remodelers at replication origins.

Many in vitro pre-RC assembly studies have focused on the molecular mechanisms regulating $\mathrm{Mcm} 2-7$ helicase loading. Since the ACS is asymmetrically located closer to the upstream nucleosome, several models posit that ORC facilitates Mcm2-7 loading in the downstream NFR (Eaton et al. 2010). First, we examined the structural chromatin changes in origin architecture that occur during preRC assembly. We identified an $~ 25$-bp Cdc6-dependent upstream or downstream extension of the origin-specific footprint in G1, accompanied by a directional expansion of the NFR (Fig. 4C). Previous in vitro DNase I hypersensitivity mapping at individual origins estimated a similar ORC footprint expansion induced by Cdc6 binding (Speck et al. 2005). A recent ORC-Cdc6 cryo-EM study suggested that ORC adopts a crescent structure along the DNA, with Cdc6 bridging the gap between the ends of the crescent where DNA both enters and exits the ORC-Cdc6 structure (Sun et al. 2012). We propose that the ORC-Cdc6 footprint extension direction is driven by chromatin or sequence-specific interactions that influence MNase accessibility to the DNA at the interface of the ORC-Cdc6 structure.

By integrating ORC and Mcm2-7 ChIP-seq data with our high-resolution chromatin profiles, we next interrogated the exact location of pre-RC assembly at replication origins across the genome (Fig. 5). ORC, as expected, mapped to the ACS within the NFR. In contrast, the Mcm2-7 complex localized either upstream of or downstream from ORC (oriented relative to the ACS). Given the upstream or downstream expansion of the ORC-dependent footprint in G1, we expected to find that the direction of the Cdc6-mediated expansion would be predictive of the Mcm2-7 double-hexamer loading side; however, the ORC 
footprint expansion side was not correlated with the Mcm2-7 loading position. We next considered whether the Mcm2-7 loading mechanism could plausibly support two different pre-RC assembly orientations. Recent cryoEM structures indicate that ORC-Cde6 undergoes a substantial conformational change from a flat crescent structure (Sun et al. 2012) to a right-handed spiral when the Cdt1-Mcm2-7 complex initially loads (Sun et al. 2013). The DNA also undergoes a dramatic transition from wrapping inside ORC-Cdc6 (Sun et al. 2012) to threading vertically through the ORC-Cdc6-Cdt1-Mcm2-7 (OCCM) intermediate structure. However, the mechanism reorienting the DNA remains unclear, and we speculate that the planar DNA within the ORC-Cdc6 complex can be rotated either clockwise or counterclockwise to occupy the OCCM inner channel. Depending on the rotation direction, the Mcm2-7 complex would be loaded either upstream of or downstream from the ACS. Primary sequence or topological constraint likely dictates the preferred DNA orientation within the OCCM structure. Alternatively, the preferential Mcm2-7 steady-state position might be chosen independently from the loading side; however, this would require substantial ORC turnover to permit Mcm2-7 sliding past ORC.

Prior work from the Bell laboratory (Lipford and Bell 2001) demonstrated the importance of precisely positioned flanking nucleosomes facilitating Mcm2-7 loading. Interestingly, we found that Mcm2-7 appears to occupy the same region of DNA as the flanking nucleosome (Fig. 5). We considered several possibilities to explain the apparent simultaneous DNA occupancy of Mcm2-7 and the flanking nucleosome (see the Results), but our data were most consistent with a nucleosome being pulled down together with the immunoprecipitated Mcm2-7 complex. Prior experiments have reported that certain $\mathrm{Mcm} 2-7$ subunits directly interact with histone H3 (Ishimi et al. 1996), and the Mcm2-7 complex mediates histone contacts with FACT and Asf1 at replication forks (Groth et al. 2007; Foltman et al. 2013). We propose that the origin-flanking nucleosomes both positionally confine and engage with Mcm2-7 in vivo to facilitate subsequent unwinding of the origin DNA in S phase.

How many Mcm2-7 double hexamers are loaded at individual origins? In theory, only one double hexamer is required to be loaded at each origin, resulting in one Mcm2-7 hexamer for each replication fork. However, it is clear from in vitro experiments that it is possible to load more than one Mcm2-7 complex on template DNA (Edwards et al. 2002; Bowers et al. 2004; Remus et al. 2009). Similarly, early work in S. cerevisiae identified a vast excess ( $\sim 20$-fold) of Mcm2-7 molecules relative to ORC in whole-cell extracts (Donovan et al. 1997). Together, these data support a model in which multiple Mcm2-7 double hexamers have the potential to load at chromosomal origins in vivo. However, our data suggest an alternative model in which only one Mcm2-7 double hexamer is loaded per origin. Specifically, we observed only one Mcm2-7 ChIP-seq peak per origin, and the Mcm2-7 complex appears to be in a tight association with the origin-flanking nucleosome. Analysis of the chromatin fragmentation distributions from the Mcm2-7 ChIP-seq yielded an immunoprecipitated DNA fragment of $\sim 210 \mathrm{bp}$, almost exactly the predicted occupancy of a histone octamer ( 150 bp) and one Mcm2-7 double hexamer ( $\sim 68 \mathrm{bp})$. Although the most direct interpretation of our data supports only one Mcm2-7 double hexamer per origin, we cannot rule out more complex models of Mcm2-7 loading (including transient and weak interactions) that may result in multiple complexes per origin.

Elegant in vitro experiments reconstituting pre-RC assembly (Speck et al. 2005; Fernández-Cid et al. 2013; Frigola et al. 2013; Sun et al. 2013) and replication initiation (Remus et al. 2009; Heller et al. 2011) have provided tremendous mechanistic insights, but assays performed on template DNA clearly lack the regulatory complexity observed at each origin in its chromosomal complex. For example, unlike the situation in vivo, preRC assembly and initiation on template DNA are not strictly dependent on origin DNA sequences (Remus et al. 2009; Gros et al. 2014). In higher eukaryotes, there is considerable plasticity in the DNA replication programs with the selection and activation of tissue- and development-specific replication origins (Rhind and Gilbert 2013). Since the genome sequence is static, regulation of these origins is very likely driven by local changes in the chromatin environment. Consistent with this hypothesis, defining features of ORC localization in Drosophila are dynamic nucleosome turnover (Deal et al. 2010) and ATPdependent chromatin remodelers (Eaton et al. 2011). Just as the expression of specific genes is fine-tuned in myriad ways, we expect that diverse chromatin-mediated mechanisms will govern the selection and activation of individual eukaryotic DNA replication origins.

\section{Materials and methods}

\section{Yeast strains}

All strains are in the W303 background and have the basic genotype ade2-1, ura3-1, his3-11,15, trp1-1, leu2-3,112, can1100; DMMy70 MATa, ade2-1, his3-11,15, leu2-3,112, can1-100, bar14::TRP, URA3::BrdU-Inc; DMMy31 MATa, ade2-1, his311,15, leu2-3,112, can1-100, orc1-161; and DMMy113 MATa, ade2-1, ura3-1, his3-11,15, leu2-3,112, can1-100, bar1s::hisG, cdc6-1 (G260D).

\section{Cell synchronization}

Wild-type and mutant (orc1-161 and cdc6-1) yeast strains were grown in rich medium for all experiments. Cells were grown at room temperature to $\mathrm{OD}_{600} 0.6$. To arrest cells at $\mathrm{G} 2 / \mathrm{M}$, nocodazole (Sigma) was added to a final concentration of $15 \mu \mathrm{g} / \mathrm{mL}$, and cells were allowed to grow for $1 \mathrm{~h}$ at $24^{\circ} \mathrm{C}$. Cultures were then shifted for an additional hour to the nonpermissive temperature, $37^{\circ} \mathrm{C}$. Cells were arrested in G1 by treatment with $\alpha$ factor (GenWay) at a concentration of $50 \mathrm{ng} / \mathrm{mL}$.

\section{Chromatin preparation}

Cells were cross-linked by adding formaldehyde to a final concentration of $1 \%$ for $30 \mathrm{~min}$ at room temperature with stirring. Formaldehyde was quenched by adding glycine to a final con- 
centration of $125 \mathrm{mM}$ for $5 \mathrm{~min}$ at room temperature with stirring. Cells were centrifuged at $2000 \mathrm{rpm}$ for $5 \mathrm{~min}$, washed with sterile water, and resuspended with $20 \mathrm{~mL}$ of buffer Z $(0.56 \mathrm{M}$ sorbitol, $50 \mathrm{mM}$ Tris at $\mathrm{pH} 7.4$, autoclaved). Next, $14 \mu \mathrm{L}$ of $\beta-\mathrm{ME}$ (United States Biological) and $0.5 \mathrm{~mL}$ of a $10 \mathrm{mg} / \mathrm{mL}$ solution of zymolyase prepared in buffer $\mathrm{Z}$ were added. Samples were incubated for $30 \mathrm{~min}$ at $24^{\circ} \mathrm{C}$ with shaking. Cells were centrifuged at $1500 \mathrm{rpm}$ for $6 \mathrm{~min}$ at $4^{\circ} \mathrm{C}$ and then resuspended in 2.5 $\mathrm{mL}$ of NP buffer ( $1 \mathrm{M}$ sorbitol, $50 \mathrm{mM} \mathrm{NaCl}, 10 \mathrm{mM}$ Tris at $\mathrm{pH}$ 7.4, $5 \mathrm{mM} \mathrm{MgCl}_{2}, 1 \mathrm{mM} \mathrm{CaCl}_{2}$, autoclaved) supplemented with $0.5 \mathrm{mM}$ spermidine, $0.007 \% \beta-\mathrm{ME}$, and $0.075 \% \mathrm{NP}-40$. Aliquots of $15 \mathrm{U} / \mu \mathrm{L}$ MNase (Worthington) were prepared to determine the best digestion conditions: $1.5-\mathrm{mL}$ tubes contained $4 \mu \mathrm{L}, 2 \mu \mathrm{L}$, $1 \mu \mathrm{L}, 0.5 \mu \mathrm{L}, 0.25 \mu \mathrm{L}$, and $0 \mu \mathrm{L}$ of MNase, to which $400 \mu \mathrm{L}$ of the resuspended cells was added. Samples were inverted to mix and digested on the benchtop for $20 \mathrm{~min}$. The reaction was halted by adding $100 \mu \mathrm{L}$ of stop buffer (5\% SDS, $50 \mathrm{mM}$ EDTA). Next, proteinase $\mathrm{K}$ was added to a $0.2 \mathrm{mg} / \mathrm{mL}$ final concentration, and the samples were inverted and then incubated overnight at $65^{\circ} \mathrm{C}$. DNA was recovered by phenol extraction and isopropanol precipitation.

\section{ChIP}

For each ChIP, a $50-\mathrm{mL}$ cell culture was grown at $30^{\circ} \mathrm{C}$ to $\mathrm{OD}_{600}$ 0.6 . For the G1-arrested samples, $\alpha$ factor (GenWay) was added to a final concentration of $50 \mathrm{ng} / \mathrm{mL}$; for the G2-arrested samples, nocodazole was added to a final concentration of $15 \mu \mathrm{g} / \mathrm{mL}$. Cells were incubated for $2 \mathrm{~h}$ at $30^{\circ} \mathrm{C}$. The chromatin extract was prepared and immunoprecipitated as previously described (Strahl-Bolsinger et al. 1997) with the following adaptations. Sonication was performed on an Ultrasonic 150T dismembrator (Fisher) at power level 030 six times for $15 \mathrm{sec}$ with incubation for $1 \mathrm{~min}$ on ice between each sonication. Samples were then centrifuged at $13,000 \mathrm{rpm}$ for $10 \mathrm{~min}$ at $4^{\circ} \mathrm{C}$ to remove cell debris. The supernatant was immunoprecipitated with $1 \mu \mathrm{L}$ of anti-ORC antibody (a gift from A. Schwacha, University of Pittsburgh) for asynchronous cells or $25 \mu \mathrm{L}$ of AS1.1 antibody (anti-Mcm2-7) for the G1-arrested cells overnight at $4^{\circ} \mathrm{C}$ with rocking. Next, $50 \mu \mathrm{L}$ of Gamma G beads (Amersham) was added and incubated for $6 \mathrm{~h}$ at $4^{\circ} \mathrm{C}$ with rocking. DNA was recovered as previously described (MacAlpine et al. 2010).

\section{Early origin activation}

A $25-\mathrm{mL}$ wild-type cell culture was grown at $30^{\circ} \mathrm{C}$ to $\mathrm{OD}_{600}$ 0.6. Next, $\alpha$ factor was added to a final concentration of $50 \mathrm{ng} /$ $\mathrm{mL}$, and cells were incubated for $2 \mathrm{~h}$. Cells were centrifuged, washed twice with sterile water, and resuspended in $25 \mathrm{~mL}$ of medium containing $0.2 \mathrm{M}$ hydroxyurea and $400 \mu \mathrm{g} / \mathrm{mL} 5$-bromodeoxyuridine (BrdU). Cells were incubated for $1.5 \mathrm{~h}$ and washed twice with sterile water. The cell pellet was quick-frozen and stored at $-80^{\circ} \mathrm{C}$. The genomic DNA was extracted, and $25 \mu \mathrm{g}$ of DNA was used in the BrdU immunoprecipitation as previously described (Ryba et al. 2011). The entire BrdU-immunoprecipitated pellet was used in the Illumina library preparation.

\section{Sequencing library preparation}

Illumina sequencing libraries of MNase-treated DNA were prepared using $500 \mathrm{ng}$ of DNA as previously described (Henikoff et al. 2011). Illumina sequencing libraries of ChIP DNA were prepared following Illumina TruSeq protocols.

\section{Sequencing read alignment to the genome}

All reads were aligned to the sacCer2/R61 version of the $S$. cerevisiae genome using Bowtie 0.12.7 (Langmead et al. 2009). The recovered sequence from all paired-end MNase reads was truncated to $20 \mathrm{bp}$ and aligned in paired-end mode using the following Bowtie parameters: -n 2 -1 20 -m 1 -k 1 -X 1000. Single-end ORC, Mcm2-7, and BrdU ChIP-seq reads were aligned with the following Bowtie parameters: -n 2 -1 30 -m 1 -k 1.

\section{Data availability}

All data have been deposited in the NCBI Sequence Read Archive (SRA) with SRA study accession number SRP041314.

\section{Acknowledgments}

We thank Jianling Zhong, Kaixuan Luo, Anirudh Natarajan, Anthony Schwacha, and members of the MacAlpine and Hartemink groups for reading the manuscript and providing critical comments and suggestions. We thank Nick Rhind for sharing unpublished data and helpful discussions. The orc1-161 and cdc6-1 mutant strains and the yeast anti-ORC antibody were gifts from Anthony Schwacha. This work was supported by the National Institutes of Health grant R01-GM104079 to D.M.M.

\section{References}

Aparicio OM, Weinstein DM, Bell SP. 1997. Components and dynamics of DNA replication complexes in S. cerevisiae: redistribution of MCM proteins and Cdc45p during S phase. Cell 91: 59-69.

Bai L, Morozov AV. 2010. Gene regulation by nucleosome positioning. Trends Genet 26: 476-483.

Bell SP, Kaguni JM. 2013. Helicase loading at chromosomal origins of replication. Cold Spring Harb Perspect Biol 5: a010124.

Bell SP, Stillman B. 1992. ATP-dependent recognition of eukaryotic origins of DNA replication by a multiprotein complex. Nature 357: 128-134.

Berbenetz NM, Nislow C, Brown GW. 2010. Diversity of eukaryotic DNA replication origins revealed by genome-wide analysis of chromatin structure. PLoS Genet 6: e1001092.

Bowers JL, Randell JCW, Chen S, Bell SP. 2004. ATP hydrolysis by ORC catalyzes reiterative $\mathrm{Mcm} 2-7$ assembly at a defined origin of replication. Mol Cell 16: 967-978.

Broach JR, Li YY, Feldman J, Jayaram M, Abraham J, Nasmyth KA, Hicks JB. 1983. Localization and sequence analysis of yeast origins of DNA replication. Cold Spring Harb Symp Quant Biol 47: 1165-1173.

Crabbé L, Thomas A, Pantesco V, De Vos J, Pasero P, Lengronne A. 2010. Analysis of replication profiles reveals key role of RFCCtf18 in yeast replication stress response. Nat Struct Mol Biol 17: 1391-1397.

Deal RB, Henikoff JG, Henikoff S. 2010. Genome-wide kinetics of nucleosome turnover determined by metabolic labeling of histones. Science 328: 1161-1164.

Diffley JFX, Cocker JH, Dowell SJ, Rowley A. 1994. Two steps in the assembly of complexes at yeast replication origins in vivo. Cell 78: 303-316.

Donovan S, Harwood J, Drury LS, Diffley JFX. 1997. Cdc6pdependent loading of $\mathrm{Mcm}$ proteins onto pre-replicative chromatin in budding yeast. Proc Natl Acad Sci 94: 5611-5616.

Eaton ML, Galani K, Kang S, Bell SP, MacAlpine DM. 2010. Conserved nucleosome positioning defines replication origins. Genes Dev 24: 748-753.

Eaton ML, Prinz JA, MacAlpine HK, Tretyakov G, Kharchenko PV, MacAlpine DM. 2011. Chromatin signatures of the Drosophila replication program. Genome Res 21: 164-174.

Edwards MC, Tutter AV, Cvetic C, Gilbert CH, Prokhorova TA, Walter JC. 2002. MCM2-7 complexes bind chromatin in a 
distributed pattern surrounding the origin recognition complex in Xenopus egg extracts. I Biol Chem 277: 3304933057.

Evrin C, Clarke P, Zech J, Lurz R, Sun J, Uhle S, Li H, Stillman B, Speck C. 2009. A double-hexameric MCM2-7 complex is loaded onto origin DNA during licensing of eukaryotic DNA replication. Proc Natl Acad Sci 106: 20240-20245.

Feng W, Collingwood D, Boeck ME, Fox LA, Alvino GM, Fangman WL, Raghuraman MK, Brewer BJ. 2006. Genomic mapping of single-stranded DNA in hydroxyurea-challenged yeasts identifies origins of replication. Nat Cell Biol 8: 148155.

Fernández-Cid A, Riera A, Tognetti S, Herrera MC, Samel S, Evrin C, Winkler C, Gardenal E, Uhle S, Speck C. 2013. An ORC/Cdc6/MCM2-7 complex is formed in a multistep reaction to serve as a platform for MCM double-hexamer assembly. Mol Cell 50: 577-588.

Foltman M, Evrin C, De Piccoli G, Jones RC, Edmondson RD, Katou Y, Nakato R, Shirahige K, Labib K. 2013. Eukaryotic replisome components cooperate to process histones during chromosome replication. Cell Reports 3: 892-904.

Frigola J, Remus D, Mehanna A, Diffley JFX. 2013. ATPasedependent quality control of DNA replication origin licensing. Nature 495: 339-343.

Gros J, Devbhandari S, Remus D. 2014. Origin plasticity during budding yeast DNA replication in vitro. EMBO I 33: 621636.

Groth A, Corpet A, Cook AJL, Roche D, Bartek J, Lukas J, Almouzni G. 2007. Regulation of replication fork progression through histone supply and demand. Science 318: 19281931.

Hartwell LH, Mortimer RK, Culotti J, Culotti M. 1973. Genetic control of the cell division cycle in yeast: V. Genetic analysis of cdc mutants. Genetics 74: 267-286.

Heller RC, Kang S, Lam WM, Chen S, Chan CS, Bell SP. 2011. Eukaryotic origin-dependent DNA replication in vitro reveals sequential action of DDK and S-CDK kinases. Cell 146: 80-91.

Henikoff JG, Belsky JA, Krassovsky K, MacAlpine DM, Henikoff S. 2011. Epigenome characterization at single base-pair resolution. Proc Natl Acad Sci 108: 18318-18323.

Ilves I, Petojevic T, Pesavento JJ, Botchan MR. 2010. Activation of the MCM2-7 helicase by association with Cdc45 and GINS proteins. Mol Cell 37: 247-258.

Ishimi Y, Ichinose S, Omori A, Sato K, Kimura H. 1996. Binding of human minichromosome maintenance proteins with histone H3. J Biol Chem 271: 24115-24122.

Kent NA, Adams S, Moorhouse A, Paszkiewicz K. 2011. Chromatin particle spectrum analysis: a method for comparative chromatin structure analysis using paired-end mode next-generation DNA sequencing. Nucleic Acids Res 39: e26.

Langmead B, Trapnell C, Pop M, Salzberg SL. 2009. Ultrafast and memory-efficient alignment of short DNA sequences to the human genome. Genome Biol 10: R25.

Leonard AC, Méchali M. 2013. DNA replication origins. Cold Spring Harb Perspect Biol 5: a010116.

Lipford JR, Bell SP. 2001. Nucleosomes positioned by ORC facilitate the initiation of DNA replication. Mol Cell 7: 21-30.

MacAlpine HK, Gordân R, Powell SK, Hartemink AJ, MacAlpine DM. 2010. Drosophila ORC localizes to open chromatin and marks sites of cohesin complex loading. Genome Res 20: 201-211.

Mantiero D, Mackenzie A, Donaldson A, Zegerman P. 2011. Limiting replication initiation factors execute the temporal programme of origin firing in budding yeast. $E M B O ~ J$ 30: 4805-4814.

Marahrens Y, Stillman B. 1992. A yeast chromosomal origin of DNA replication defined by multiple functional elements. Science 255: 817-823.

Mavrich TN, Ioshikhes IP, Venters BJ, Jiang C, Tomsho LP, Qi J, Schuster SC, Albert I, Pugh BF. 2008. A barrier nucleosome model for statistical positioning of nucleosomes throughout the yeast genome. Genome Res 18: 1073-1083.

McGuffee SR, Smith DI, Whitehouse I. 2013. Quantitative, genome-wide analysis of eukaryotic replication initiation and termination. Mol Cell 50: 123-135.

Müller CA, Hawkins M, Retkute R, Malla S, Wilson R, Blythe MJ, Nakato R, Komata M, Shirahige K, de Moura APS, et al. 2014. The dynamics of genome replication using deep sequencing. Nucleic Acids Res 42: e3.

Nieduszynski CA, Hiraga SI, Ak P, Benham CJ, Donaldson AD. 2007. OriDB: a DNA replication origin database. Nucleic Acids Res 35: D40-D46.

Onishi M, Liou GG, Buchberger JR, Walz T, Moazed D. 2007. Role of the conserved Sir3-BAH domain in nucleosome binding and silent chromatin assembly. Mol Cell 28: 10151028.

Pasero P, Duncker BP, Schwob E, Gasser SM. 1999. A role for the Cdc7 kinase regulatory subunit Dbf4p in the formation of initiation-competent origins of replication. Genes Dev 13: 2159-2176.

Perkins G, Diffley JFX. 1998. Nucleotide-dependent prereplicative complex assembly by Cdc6p, a homolog of eukaryotic and prokaryotic clamp-loaders. Mol Cell 2: 23-32.

Raghuraman MK, Winzeler EA, Collingwood D, Hunt S, Wodicka L, Conway A, Lockhart DJ, Davis RW, Brewer BJ, Fangman WL. 2001. Replication dynamics of the yeast genome. Science 294: 115-121.

Remus D, Diffley JFX. 2009. Eukaryotic DNA replication control: lock and load, then fire. Curr Opin Cell Biol 21: 771-777.

Remus D, Beuron F, Tolun G, Griffith JD, Morris EP, Diffley JFX. 2009. Concerted loading of Mcm2-7 double hexamers around DNA during DNA replication origin licensing. Cell 139: 719-730.

Rhind N, Gilbert DM. 2013. DNA replication timing. Cold Spring Harb Perspect Biol 5: a010132.

Ryba T, Battaglia D, Pope BD, Hiratani I, Gilbert DM. 2011. Genome-scale analysis of replication timing: from bench to bioinformatics. Nat Protoc 6: 870-895.

Schmiedeberg L, Skene P, Deaton A, Bird A. 2009. A temporal threshold for formaldehyde crosslinking and fixation. PLoS ONE 4: e4636.

Shimada K, Oma Y, Schleker T, Kugou K, Ohta K, Harata M, Gasser SM. 2008. Ino80 chromatin remodeling complex promotes recovery of stalled replication forks. Curr Biol 18: 566-575.

Simpson RT. 1990. Nucleosome positioning can affect the function of a cis-acting DNA element in vivo. Nature 343: 387-389.

Speck C, Chen Z, Li H, Stillman B. 2005. ATPase-dependent cooperative binding of ORC and Cdc6 to origin DNA. Nat Struct Mol Biol 12: 965-971.

Strahl-Bolsinger S, Hecht A, Luo K, Grunstein M. 1997. SIR2 and SIR4 interactions differ in core and extended telomeric heterochromatin in yeast. Genes Dev 11: 83-93.

Sun J, Kawakami H, Zech J, Speck C, Stillman B, Li H. 2012. Cdc6-induced conformational changes in ORC bound to origin DNA revealed by cryo-electron microscopy. Structure 20: $534-544$. 
Belsky et al.

Sun J, Evrin C, Samel SA, Fernández-Cid A, Riera A, Kawakami H, Stillman B, Speck C, Li H. 2013. Cryo-EM structure of a helicase loading intermediate containing ORC-Cdc6-Cdt1MCM2-7 bound to DNA. Nat Struct Mol Biol 20: 944-951.

Szilard RK, Jacques PE, Laramée L, Cheng B, Galicia S, Bataille AR, Yeung M, Mendez M, Bergeron M, Robert F, et al. 2010. Systematic identification of fragile sites via genome-wide location analysis of $\gamma$-H2AX. Nat Struct Mol Biol 17: 299305.

Tanaka S, Nakato R, Katou Y, Shirahige K, Araki H. 2011. Origin association of Sld3, Sld7, and Cde45 proteins is a key step for determination of origin-firing timing. Curr Biol 21: 2055-2063.

Teytelman L, Thurtle DM, Rine J, van Oudenaarden A. 2013. Highly expressed loci are vulnerable to misleading ChIP localization of multiple unrelated proteins. Proc Natl Acad Sci 110: 18602-18607.

Vincent JA, Kwong TJ, Tsukiyama T. 2008. ATP-dependent chromatin remodeling shapes the DNA replication landscape. Nat Struct Mol Biol 15: 477-484.

Wu PYJ, Nurse P. 2009. Establishing the program of origin firing during S phase in fission yeast. Cell 136: 852-864.

Wyrick JJ, Aparicio JG, Chen T, Barnett JD, Jennings EG, Young RA, Bell SP, Aparicio OM. 2001. Genome-wide distribution of ORC and MCM proteins in S. cerevisiae: high-resolution mapping of replication origins. Science 294: 2357-2360.

Xu W, Aparicio JG, Aparicio OM, Tavaré S. 2006. Genome-wide mapping of ORC and $\mathrm{Mcm} 2 \mathrm{p}$ binding sites on tiling arrays and identification of essential ARS consensus sequences in S. cerevisiae. BMC Genomics 7: 276.

Yabuki N, Terashima H, Kitada K. 2002. Mapping of early firing origins on a replication profile of budding yeast. Genes Cells 7: 781-789.

Zhang Y, Moqtaderi Z, Rattner BP, Euskirchen G, Snyder M, Kadonaga JT, Liu XS, Struhl K. 2009. Intrinsic histone-DNA interactions are not the major determinant of nucleosome positions in vivo. Nat Struct Mol Biol 16: 847-852. 


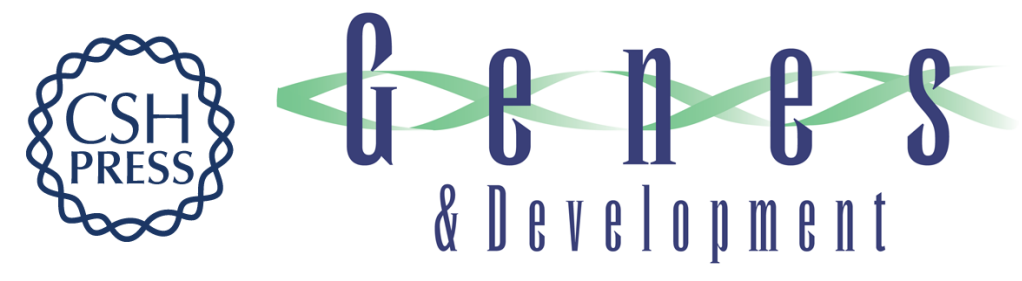

\section{Genome-wide chromatin footprinting reveals changes in replication origin architecture induced by pre-RC assembly}

Jason A. Belsky, Heather K. MacAlpine, Yoav Lubelsky, et al.

Genes Dev. 2015, 29:

Access the most recent version at doi:10.1101/gad.247924.114

\section{Supplemental http://genesdev.cshlp.org/content/suppl/2015/01/15/29.2.212.DC1 \\ Material}

References This article cites 61 articles, 24 of which can be accessed free at:

http://genesdev.cshlp.org/content/29/2/212.full.html\#ref-list-1

Creative This article is distributed exclusively by Cold Spring Harbor Laboratory Press for the first

Commons six months after the full-issue publication date (see

License http://genesdev.cshlp.org/site/misc/terms.xhtml). After six months, it is available under a Creative Commons License (Attribution-NonCommercial 4.0 International), as described at http://creativecommons.org/licenses/by-nc/4.0/.

Email Alerting Receive free email alerts when new articles cite this article - sign up in the box at the top Service right corner of the article or click here.

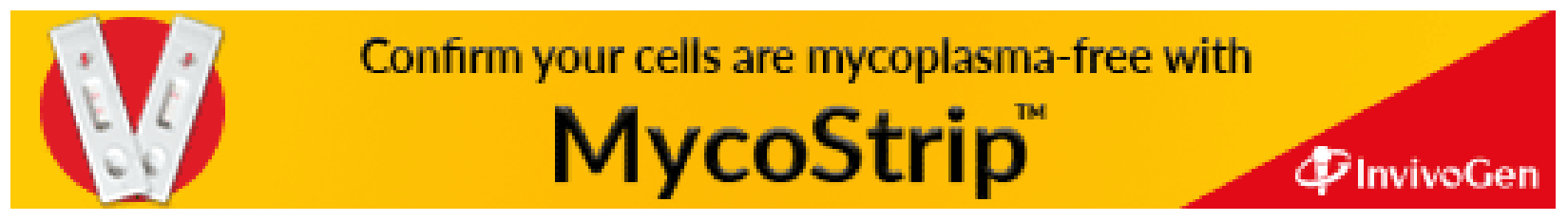

